\title{
Concentration of 8-isoprostanes in the exhaled breath condensate as a marker of oxidative stress in patients with type 1 diabetes
}

The authors declare no finacial disclosure

\begin{abstract}
Introduction: Type 1 diabetes is an insulin deficiency-based chronic disease. It leads to the development of hyperglycaemia, which plays a key role in the initiation and progression of tissue damage in patients with diabetes. This mostly results from oxidative stress, whose increased severity is observed in this group of patients. Increased levels of 8-isoprostanes are seen in many inflammatory diseases, including asthma, COPD and cystic fibrosis. In these diseases it was demonstrated exhaled breath condensate $(E B C)$ is useful for extracting material for markers of oxidative stress, including 8-isoprostanes. The purpose of this study was to assess the severity of oxidative stress measured with 8-isoprostane concentrations in the exhaled breath condensate in healthy subjects and in patients with type 1 diabetes with and without vascular complications.

Material and methods: 33 patients assigned to the control group, type 1 diabetes without complications group and type 1 diabetes group with advanced complications were included in the study. Retinopathy, nephropathy or neuropathy have been reported as a criterion distinguishing between complicated and uncomplicated diabetes. EBC was obtained for each subject. 8-isoprostane concentrations were determined in serum and EBC by ELISA.

Results and conclusions: Mean $( \pm S D$ ) blood levels of 8-isoprostane in patients with type 1 diabetes mellitus without complications and those with type 1 diabetes with advanced complications were significantly higher compared to the control group (178.17 [135.73] vs. 183.34 [200.41] vs. 47.13 [25.20] pg/ml; $p<0.05)$. The mean ( \pm SD) concentration of 8-isoprostane in EBC was lower in diabetic patients with type 1 diabetes with advanced complications than in patients with type 1 diabetes without advanced complications and in the control group (8.32 [4.60] vs. 19.13 [22.35] vs. 28.17 [35.11] pg/ml; $p<0.05$ ). Measurement of 8-isoprostanes in the EBC in patients with type 1 diabetes does not appear to be a good diagnostic tool for monitoring the activity of oxidative stress in these patients.
\end{abstract}

Key words: 8-isoprostanes, exhaled breath condensate, oxidative stress, type 1 diabetes

Adv Respir Med. 2018; 86: 3-6

\section{Introduction}

Type 1 diabetes is an insulin deficiency-based chronic disease. It leads to the development of hyperglycaemia, which plays a key role in the initiation and progression of tissue damage in patients with diabetes [1]. This mostly results from oxidative stress, whose increased severity is observed in this group of patients [2].
Oxidative stress is a phenomenon present in properly functioning living organisms. Its excessive exacerbation can lead to adverse effects such as damage to cellular structures and DNA, and may consequently affect the development of various diseases, including cancer [3]. One of the tools used to determine the severity of oxidative stress involves the measurement of the 8-isoprostane concentration that results from non-enzymat-

Address for correspondence: Michał Poznański, Department of General and Oncological Pneumonology, First Chair of Internal Diseases, Medical University of Lodz Kopcińskiego 22, 90-153 Łódź, e-mail: michal.poznanski@umed.lodz.pl DOI: 10.5603/ARM.2018.0002

Received: 11.10.2017

Copyright (C) 2018 PTChP

ISSN 2451-4934 
ic lipid peroxidation accompanied by free radicals $[4,5]$. Increased levels of 8 -isoprostanes are seen in many inflammatory diseases, including asthma [6], COPD [7] and cystic fibrosis [8]. In these diseases In these demonstrated, that EBC is useful for extracting material for markers of oxidative stress, including 8-isoprostanes. The ease of obtaining material during EBC testing and non-invasive and extensive safety profiles continue to encourage further exploration of this research method.

The purpose of this study was to assess the severity of oxidative stress measured with 8-isoprostane concentrations in the exhaled breath condensate in healthy subjects and in patients with and without vascular complications.

According to the author's knowledge, this is the first work in which oxidative stress in type 1 diabetes was measured by the concentration of 8-isoprostanes in the exhaled breath condensate.

\section{Material and methods}

Thirty three patients assigned to the control group, type 1 diabetes without complications group and type 1 diabetes group with advanced complications were included in the study. The subjects included in the study were recruited from the Diabetes Outpatient Clinic of the University Clinical Hospital No. 1 in Łódź.

The control group consisted of 12 healthy persons randomly selected among the staff of the University Clinical Hospital No. 1 in Łódź.

The control group excluded patients with:

- Any type of diabetes

- Incorrect glucose metabolism, defined as abnormal fasting plasma glucose

- Smoking history, chronic pulmonary disease and other chronic diseases and metabolic disorders

- Familial type 1 diabetes

The criteria for inclusion in the study included the following:
- Documented type 1 diabetes with a duration of not less than 15 years.

- Incorrect metabolic rate of diabetes, assessed basing on 3 historical concentrations of glycated haemoglobin (HbA1c).

- Diabetes treated with a model of intensive insulin therapy.

- Non-smoking.

- No history of chronic lung disease.

The research was approved by the Bioethics Committee at the Medical University of Łódź. All participants have signed a conscious and voluntary agreement to participate in the study. Once selected, anthropometric parameters (body mass and height) were determined. Retinopathy, nephropathy or neuropathy have been reported as a criterion distinguishing between complicated and uncomplicated diabetes. The presence of diabetic retinopathy was verified on the basis of ophthalmic examination of the fundus by means of the Volk 90D lens, after the pupil dilatation with the atropine, always performed by the same physician.

The value of the albumin/creatinine index (ACR) was calculated for each subject as the basis of renal complications. Basing on the results thereof, the patient was assigned to the no renal complication group (ACR $<30 \mathrm{mg} / \mathrm{g}$ ) or to advanced renal disease (ACR $\geq 30 \mathrm{mg} / \mathrm{g}$ ) [9].

Medical history was used to assess neuropathy; a temperature and touch sensation study, and a tendon reflex assessment were performed.

The following laboratory tests: morphology, lipidogram, urea, creatinine, electrolytes, alanine aminotransferase and aspartate aminotransferase were made for a more accurate assessment of health status. All studies were performed at the Hospital Laboratory of the University Hospital No. 1 in Łódź. On the same day, EBC was obtained for each patient by means of the EcoScreen condenser (Jaeger). Each of the subjects was asked to calmly breathe for 10 minutes using a condenser.

Table 1. Characteristics of groups

\begin{tabular}{lccc}
\hline & $\begin{array}{c}\text { Patients with no complications } \\
(\mathbf{n}=\mathbf{1 0})\end{array}$ & $\begin{array}{c}\text { Patients with complications } \\
(\mathbf{n}=\mathbf{1 1})\end{array}$ & $\begin{array}{c}\text { Control } \\
(\mathbf{n}=\mathbf{1 2})\end{array}$ \\
\hline Sex (M/F) & $3 / 7$ & $5 / 6$ & $2 / 10$ \\
Age median (years/min.-max.) & $31 / 19-55$ & $49 / 26-70$ & $34 / 25-60$ \\
Mean BMI/SD & $24.0 / 3.4$ & $26.6 / 4.5$ & $24.4 / 1.6$ \\
Median of disease duration & $21 / 15-25$ & $24 / 20-36$ & $\mathrm{~N} / \mathrm{A}$ \\
(years/min.-max.) & & & $5.5 / 0.2$ \\
Mean $\mathrm{HbA}_{1} \mathrm{c}$ (\%/SD) & $8.2 / 1.6$ & $8.5 / 1.4$ & \\
\hline
\end{tabular}

$\mathrm{BMI}$ - body mass index; SD - standard deviation 
The resulting material was frozen at $-80^{\circ} \mathrm{C}$ for further analysis.

8-isoprostane concentrations were determined in serum and EBC by ELISA using 8-isoprostane ELISA Kit (Cayman Chemical; Ann Arbor, MI, USA). The results were read by means of EL808 IU (BioTek; Winooski, VT, USA).

STATISTICA version 12.0 (StatSoft Inc.) license number JPZP601E504326AR-9 was used to perform statistical calculations. The normality of distribution of empirical variables was assessed by graphic analysis of histograms drawn from collected data and by the Shapiro-Wilk test. This method was used for the part of the data for which the normality of the distribution has been demonstrated, and the other requirements used for the analysis of variance $\left(\mathrm{ANOVA}^{\mathrm{TM}}\right)$ have been met. Data that did not show normal distribution even after transformations were analysed using the Kruskal-Wallis test. For multiple comparisons, the Least Significant Difference (LSD) test was used. Whenever the calculated probability coefficient $\mathrm{p}$ was less than 0.05 , the result was considered statistically significant.

\section{Results}

Mean ( \pm SD) blood levels of 8-isoprostane in patients with type 1 diabetes mellitus without complications and those with type 1 diabetes with advanced complications were significantly higher compared to the control group (178.17 [135.73] vs. $183.34[200.41]$ vs. $47.13[25.20] \mathrm{pg} / \mathrm{ml}$; $\mathrm{p}<0.05)-$ graphic illustration of the result is shown in Figure 1.

The mean $( \pm$ SD) concentration of 8-isoprostane in EBC was lower in diabetic patients with type 1 diabetes with advanced complications than in patients with type 1 diabetes without advanced complications and in the control group (8.32 [4.60] vs. 19.13 [22.35]. 28.17 [35.11] pg/ml; $\mathrm{p}<0.05)$ - graphic illustration of the result is presented in Figure 2. We did not manage to demonstrate the correlation of the variables tested.

\section{Discussion}

The decision to choose type 1 diabetes patients was not accidental. This is a group of patients particularly exposed to the development of complications from an early age, and angiopathy affects all vessels, including those in the pulmonary circulation. Therefore, the usefulness of non-invasive methods (EBC) in assessing the severity of oxidative stress in this group of patients seems to be an interesting issue.

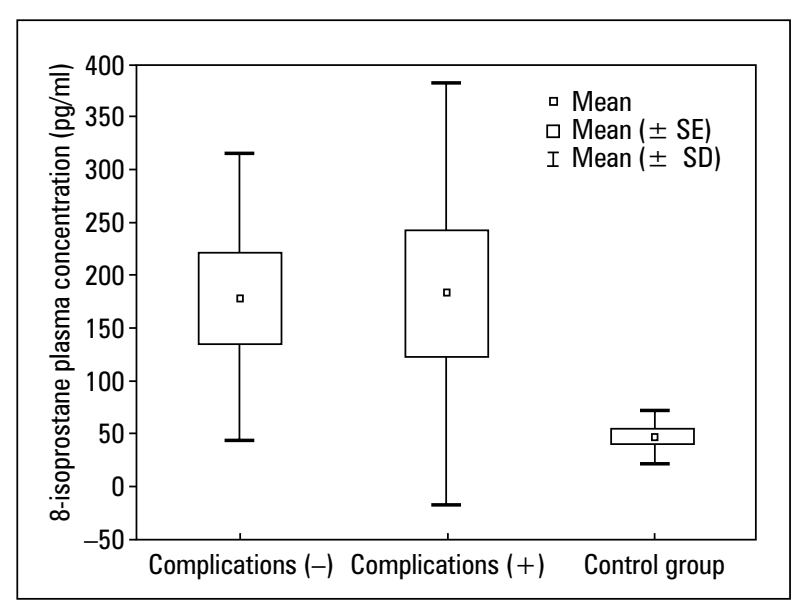

Figure 1. Box and whisker diagram of mean plasma concentrations of 8-isoprostanes in patients

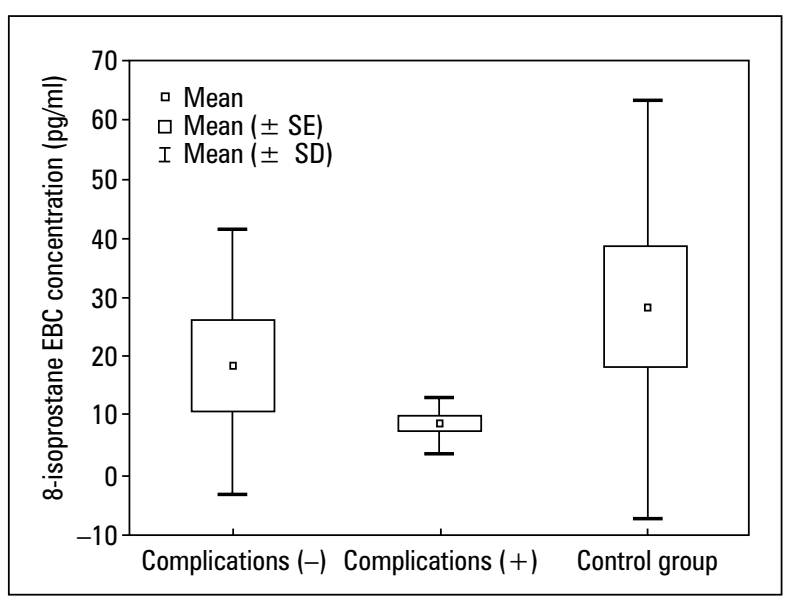

Figure 2. Box and whisker diagram of mean plasma concentrations of 8-isoprostanes in EBC

The mean concentration of 8-isoprostanes was the highest in plasma in type 1 diabetic patients with developed complications. Lower levels of this marker were characteristic of a group with no developed complications. The lowest values were observed in the control group. These results appear to be consistent with the intuitively expected values, providing a biochemical evidence that the 8-isoprostane concentration is significantly higher in the plasma of inflammatory patients. Similar observations come from, among others, the work of Vessby [10].

Surprising results were obtained from the exhaled breath condensate analysis, where the highest concentrations of 8-isoprostanes were observed in the control group. This suggests that there may be no localised oxidative stress in the altered pulmonary vascular endothelium. This 
simple explanation, however, is in contradiction with the observed increase in the concentration of methyl nitrate in the EBC and with the increase in glucose levels in blood in patients with type 1 diabetes [11]. A similar observation suggesting an increase in oxidation and reduction in pulmonary vessels in diabetes was demonstrated in the animal model. It has been observed that the concentration of ethane, which is one of the lipid peroxidation products, is higher in the air exhaled in both acute and chronic hyperglycaemic rats [12]. The mechanism of diffusion of 8-isoprostanes from the blood into the lung follicles is not fully understood. Certainly, any structural change in this pathway may result in changes in EBC compound concentrations, which, in the context of observed basophilic thickening of the membrane in patients with diabetes [1,13-18], seems to be a reasonable explanation for our observations.

Because the research problem has not been studied so far, the results we have obtained need to be confirmed in subsequent scientific studies.

\section{Conclusions}

1. Type 1 diabetes is accompanied by increased oxidative stress manifested by increased lipid peroxidation, measured by plasma concentrations of 8-isoprostanes.

2. The highest plasma levels of 8-isoprostanes are noted in patients with type 1 diabetes with advanced complications.

3. The concentration of 8-isoprostanes in the EBC of patients with type 1 diabetes is lower compared to healthy subjects.

4. Measurement of 8-isoprostanes in the EBC in patients with type 1 diabetes does not appear to be a good diagnostic tool for monitoring the activity of oxidative stress in these patients.

\section{Conflict of interest}

The authors declare no conflict of interest.

\section{References:}

1. Devries JH, Snoek FJ, Heine RJ. Persistent poor glycaemic control in adult Type 1 diabetes. A closer look at the problem. Diabet Med. 2004; 21(12): 1263-1268, doi: 10.1111/j. 1464-5491.2004.01386.x, indexed in Pubmed: 15569126.
2. Wentholt IME, Kulik W, Michels RPJ, et al. Glucose fluctuations and activation of oxidative stress in patients with type 1 diabetes. Diabetologia. 2008; 51(1): 183-190, doi: 10.1007/ s00125-007-0842-6, indexed in Pubmed: 17994218.

3. Kawanishi S, Ohnishi S, Ma N, et al. Crosstalk between DNA Damage and Inflammation in the Multiple Steps of Carcinogenesis. Int J Mol Sci. 2017; 18(8), doi: 10.3390/ijms18081808, indexed in Pubmed: 28825631.

4. Dizdaroglu M, Jaruga P, Birincioglu M, et al. Free radical-induced damage to DNA: mechanisms and measurement. Free Radic Biol Med. 2002; 32(11): 1102-1115, indexed in Pubmed: 12031895.

5. de Zwart LL, Meerman JH, Commandeur JN, et al. Biomarkers of free radical damage applications in experimental animals and in humans. Free Radic Biol Med. 1999; 26(1-2): 202-226, indexed in Pubmed: 9890655.

6. Montuschi P, Corradi M, Ciabattoni G, et al. Increased 8-isoprostane, a marker of oxidative stress, in exhaled condensate of asthma patients. Am J Respir Crit Care Med. 1999; 160(1): 216-220, doi: 10.1164/ajrccm.160.1.9809140, indexed in Pubmed: 10390403

7. Carpagnano GE, Kharitonov SA, Foschino-Barbaro MP, et al. Supplementary oxygen in healthy subjects and those with COPD increases oxidative stress and airway inflammation. Thorax. 2004; 59(12): 1016-1019, doi: 10.1136/ thx.2003.020768, indexed in Pubmed: 15563698.

8. Kharitonov SA, Barnes PJ. Biomarkers of some pulmonary diseases in exhaled breath. Biomarkers. 2002; 7(1): 1-32, doi: 10.1080/13547500110104233, indexed in Pubmed: 12101782.

9. Bakker AJ. Detection of microalbuminuria. Receiver operating characteristic curve analysis favors albumin-to-creatinine ratio over albumin concentration. Diabetes Care. 1999; 22(2): 307-313, indexed in Pubmed: 10333950.

10. Vessby J, Basu S, Mohsen R, et al. Oxidative stress and antioxidant status in type 1 diabetes mellitus. J Intern Med. 2002; 251(1): 69-76, indexed in Pubmed: 11851867.

11. Novak BJ, Blake DR, Meinardi S, et al. Exhaled methyl nitrate as a noninvasive marker of hyperglycemia in type 1 diabetes. Proc Natl Acad Sci U S A. 2007; 104(40): 15613-15618, doi: 10.1073/pnas.0706533104, indexed in Pubmed: 17895380.

12. Habib MP, Dickerson FD, Mooradian AD. Effect of diabetes, insulin, and glucose load on lipid peroxidation in the rat. Metabolism. 1994; 43(11): 1442-1445, indexed in Pubmed: 7968601.

13. Vracko R, Thorning D, Huang TW. Basal lamina of alveolar epithelium and capillaries: quantitative changes with aging and in diabetes mellitus. Am Rev Respir Dis. 1979; 120(5): 973-983, doi: 10.1164/arrd.1979.120.5.973, indexed in Pubmed: 507532

14. Weynand B, Jonckheere A, Frans A, et al. Diabetes mellitus induces a thickening of the pulmonary basal lamina. Respiration. 1999; 66(1): 14-19, doi: 10.1159/000029331, indexed in Pubmed: 9973685.

15. Goldman MD. Lung dysfunction in diabetes. Diabetes Care. 2003; 26(6): 1915-1918, indexed in Pubmed: 12766133.

16. Ardigo D, Valtuena S, Zavaroni I, et al. Pulmonary Complications in Diabetes Mellitus: The Role of Glycemic Control. Current Drug Target -Inflammation \& Allergy. 2004; 3(4): 455-458, doi: 10.2174/1568010042634488.

17. Hsia CCW, Raskin P. The diabetic lung: relevance of alveolar microangiopathy for the use of inhaled insulin. Am J Med. 2005; 118(3): 205-211, doi: 10.1016/j.amjmed.2004.09.019, indexed in Pubmed: 15745714.

18. Kuitert LME. The lung in diabetes - yet another target organ? Chron Respir Dis. 2008; 5(2): 67-68, doi 10.1177/1479972308091408, indexed in Pubmed: 18539718. 